\title{
One More Bit Is Enough
}

\author{
Yong Xia* Lakshminarayanan Subramanian+ \\ * ECSE Department \\ Rensselaer Polytechnic Institute \\ \{xiay@alum,shivkuma@ecse\}.rpi.edu
}

\author{
Ion Stoica ${ }^{+}$Shivkumar Kalyanaraman* \\ + EECS Department \\ University of California, Berkeley \\ \{lakme, istoica\}@cs.berkeley.edu
}

\begin{abstract}
Achieving efficient and fair bandwidth allocation while minimizing packet loss in high bandwidth-delay product networks has long been a daunting challenge. Existing endto-end congestion control (e.g., TCP) and traditional congestion notification schemes (e.g., TCP+AQM/ECN) have significant limitations in achieving this goal. While the recently proposed XCP protocol addresses this challenge, XCP requires multiple bits to encode the congestion-related information exchanged between routers and end-hosts. Unfortunately, there is no space in the IP header for these bits, and solving this problem involves a non-trivial and timeconsuming standardization process.

In this paper, we design and implement a simple, lowcomplexity protocol, called Variable-structure congestion Control Protocol (VCP), that leverages only the existing two ECN bits for network congestion feedback, and yet achieves comparable performance to XCP, i.e., high utilization, low persistent queue length, negligible packet loss rate, and reasonable fairness. On the downside, VCP converges significantly slower to a fair allocation than XCP. We evaluate the performance of $\mathrm{VCP}$ using extensive ns2 simulations over a wide range of network scenarios. To gain insight into the behavior of VCP, we analyze a simple fluid model, and prove a global stability result for the case of a single bottleneck link shared by flows with identical round-trip times.
\end{abstract}

\section{Categories and Subject Descriptors}

C.2.2 [Computer-Communication Networks]: Network Protocols

General Terms

Algorithms, Design, Experimentation, Performance, Theory

Keywords

Congestion Control, Protocol, TCP, AQM, ECN, XCP

\section{INTRODUCTION}

The Additive-Increase-Multiplicative-Decrease (AIMD) [10] congestion control algorithm employed by TCP [25] is known

Permission to make digital or hard copies of all or part of this work for personal or classroom use is granted without fee provided that copies are not made or distributed for profit or commercial advantage and that copies bear this notice and the full citation on the first page. To copy otherwise, to republish, to post on servers or to redistribute to lists, requires prior specific permission and/or a fee.

SIGCOMM'05, August 22-26, 2005, Philadelphia, Pennsylvania, USA.

Copyright 2005 ACM 1-59593-009-4/05/0008 ...\$5.00. to be ill-suited for high Bandwidth-Delay Product (BDP) networks. With rapid advances in the deployment of very high bandwidth links in the Internet, the need for a viable replacement of TCP in such environments has become increasingly important.

Several research efforts have proposed different approaches for this problem, each with their own strengths and limitations. These can be broadly classified into two categories: end-to-end and network feedback based approaches. Pure end-to-end congestion control schemes such as HighSpeed TCP [15], FAST [31] and BIC [67, 59], although being attractive solutions for the short-term (due to a lesser deployment barrier), may not be suitable as long-term solutions. Indeed, in high BDP networks, using loss and/or delay as the only congestion signal(s) poses fundamental limitations on achieving high utilization and fairness while maintaining low bottleneck queue length and minimizing congestioninduced packet drop rate. HighSpeed TCP illustrates the limitations of loss-based approaches in high bandwidth optical links with very low bit-error rates [15]. Similarly, Bullot and Cottrell show that delay-based approaches are highly sensitive to minor delay variations [7], a common case in today's Internet.

To address some of the limitations of end-to-end congestion control schemes, many researchers have proposed the use of explicit network feedback. However, while traditional congestion notification feedback schemes such as $\mathrm{TCP}+\mathrm{AQM} / \mathrm{ECN}$ proposals $[18,2,42,57]$ are successful in reducing the loss rate and the queue size in the network, they still fall short in achieving high utilization in high BDP networks [24, 49, 35]. XCP [35] addresses this problem by having routers estimate the fair rate and send this rate back to the senders. Congestion control schemes that use explicit rate feedback have been also proposed in the context of the ATM Available Bit Rate (ABR) service [40, 9, 33, 27, 34]. However, these schemes are hard to deploy in today's Internet as they require a non-trivial number of bits to encode the rate, bits which are not available in the IP header.

In this paper, we show that it is possible to approximate XCP's performance in high BDP networks by leveraging only the two ECN bits (already present in the IP header) to encode the congestion feedback. The crux of our algorithm, called Variable-structure congestion Control Protocol (VCP), is to dynamically adapt the congestion control policy as a function of the level of congestion in the network. With VCP, each router computes a load factor [27], and uses this factor to classify the level of congestion into three regions: low-load, high-load and overload [28]. The 
router encodes the level of congestion in the ECN bits. As with ECN, the receiver sends the congestion information to the sender via acknowledgement (ACK) packets. Based on the load region reported by the network, the sender uses one of the following policies: Multiplicative Increase (MI) in the low-load region, Additive Increase (AI) in the high-load region, and Multiplicative Decrease (MD) in the overload region. By using MI in the low-load region, flows can exponentially ramp up their bandwidth to improve network utilization. Once high utilization is attained, AIMD provides long-term fairness amongst the competing flows.

Using extensive packet-level ns2 [52] simulations that cover a wide range of network scenarios, we show that VCP can approximate the performance of XCP by achieving high utilization, low persistent queue length, negligible packet drop rate and reasonable fairness. One limitation of VCP (as is the case for other end-host based approaches including TCP and $\mathrm{TCP}+\mathrm{AQM})$ is that it converges significantly slower to a fair allocation than XCP.

To better understand the VCP's behavior, we use a simple fluid model that approximates the behavior of VCP. For the case of a single bottleneck link shared by flows with identical round-trip delays, we prove that the model asymptotically achieves global stability independent of the link capacity, the feedback delay and the number of flows. For more general multiple-bottleneck topologies, we show that the equilibrium rate allocation of this model is max-min fair [4]. While this model may not accurately reflect VCP's dynamics, it does reinforce the stability and fairness properties that we observe in our simulations and provides a good theoretical grounding for VCP.

From a practical point of view VCP has two advantages. First, VCP does not require any modifications to the IP header since it can reuse the two ECN bits in a way that is compatible with the ECN proposal [57]. Second, it is a simple protocol with low algorithmic complexity. The complexity of VCP's end-host algorithm is similar to that of TCP. The router algorithm maintains no per-flow state, and it has very low computation complexity. We believe that these benefits largely offset VCP's limitation of having a much slower fairness convergence speed than XCP.

The rest of the paper is organized as follows. In Section 2, we describe the guidelines that motivate the design of VCP and in Section 3, we provide a detailed description of VCP. In Section 4, we evaluate the performance of VCP using extensive simulations. In Section 5, we develop a fluid model that approximates VCP's behavior and characterize its stability, fairness and convergence properties (with the detailed proofs presented in a technical report [66]). Section 6 addresses concerns on the stability of VCP under heterogeneous delays and the influence of switching between MI, AI and MD on efficiency and fairness. We review related work in Section 7 and summarize our findings in Section 8.

\section{FOUNDATIONS}

In this section, we first review why XCP scales to high BDP networks while TCP+AQM does not. Then, we present two guidelines that form the basis of the VCP design.

\subsection{Why XCP outperforms TCP+AQM?}

There are two main reasons of why TCP does not scale to high BDP networks. First, packet loss is a binary congestion signal that conveys no information about the degree of congestion. Second, due to stability reasons, relying only on packet loss for congestion indication requires TCP to use a conservative window increment policy and an aggressive window decrement policy $[25,35]$. In high BDP networks, every loss event forces a TCP flow to perform an MD, followed by the slow convergence of the AI algorithm to reach high utilization. Since the time for each individual AIMD epoch is proportional to the per-flow BDP, TCP flows remain in low utilization regions for prolonged periods of time thereby resulting in poor link utilization. Using AQM/ECN in conjunction with TCP does not solve this problem since the (one-bit) ECN feedback, similar to a packet loss, is not indicative of the degree of congestion either.

$\mathrm{XCP}$ addresses this problem by precisely measuring the fair share of a flow at a router and providing explicit rate feedback to end-hosts. One noteworthy aspect of XCP is the decoupling of efficiency control and fairness control at each router. XCP uses MIMD to control the flow aggregate and converge exponentially fast to any available bandwidth and uses AIMD to fairly allocate the bandwidth among competing flows. XCP, however, requires multiple bits in the packet header to carry bandwidth allocation information $(\Delta c w n d)$ from network routers to end-hosts, and congestion window (cwnd) and Round-Trip Time (RTT) information ( $r t t)$ from the end-hosts to the network routers.

\subsection{Design Guidelines for VCP}

The main goal of our work is to develop a simple congestion control mechanism that can scale to high BDP networks. By "simple" we mean an AQM-style approach where routers merely provide feedback on the level of network congestion, and end-hosts perform congestion control actions using this feedback. Furthermore, to maintain the compatibility with the existing IP header format, we restrict ourselves to using only two bits to encode the congestion information. To address these challenges, our solution builds around two design guidelines:

\section{\#1, Decouple efficiency control \& fairness control.}

Like XCP, VCP decouples efficiency and fairness control. However, unlike $\mathrm{XCP}$ where routers run the efficiency and fairness control algorithms and then explicitly communicate the rate to end-hosts, VCP routers compute only a congestion level, and end-hosts run one of the two algorithms as a function of the congestion level. More precisely, VCP classifies the network utilization into different utilization regions [28] and determines the controller that is suitable for a given region. Efficiency and fairness have different levels of relative importance in different utilization regions. When network utilization is low, the goal of VCP is to improve efficiency more than fairness. On the other hand, when utilization is high, VCP accords higher priority to fairness than efficiency. By decoupling these two issues, end-hosts have only a single objective in each region and thus need to apply only one congestion response. For example, one such choice of congestion response, which we use in VCP, is to perform $\mathrm{MI}$ in low utilization regions for improving efficiency, and to apply AIMD in high utilization regions for achieving fairness. The goal then is to switch between these two congestion responses depending on the level of network utilization.

\section{\#2, Use link load factor as the congestion signal.}

$\mathrm{XCP}$ uses spare bandwidth (the difference between capac- 


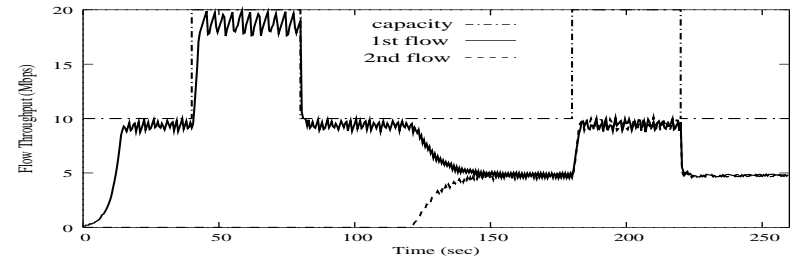

Figure 1: The throughput dynamics of two flows of the same RTT (80ms). They share one bottleneck with the capacity bouncing between 10Mbps and 20Mbps. This simple example unveils VCP's potential to quickly track changes in available bandwidth (with load-factor guided MIMD) and thereafter achieve a fair bandwidth allocation (with AIMD).

ity and demand) as a measure of the degree of congestion. In $\mathrm{VCP}$, we use load factor as the congestion signal, i.e., the relative ratio of demand and capacity [27].

While the load factor conveys less information than spare bandwidth, the fact that the load factor is a scale-free parameter allows us to encode it using a small number of bits without much loss of information. In this paper, we show that a two-bit encoding of the load factor is sufficient to approximate XCP's performance. Note that in comparison to binary congestion signals such as loss and one-bit ECN, the load factor conveys more information about the degree of network congestion.

\subsection{A Simple Illustration}

In this subsection, we give a high level description of $\mathrm{VCP}$ using a simple example. A detailed description of VCP is presented in Section 3. Periodically, each router measures the load factor for its output links and classifies the load factor into three utilization regions: low-load, high-load or overload. Each router encodes the utilization regions in the two ECN bits in the IP header of each data packet. In turn, the receiver sends back this information to the sender via the ACK packets. Depending on this congestion information, the sender applies different congestion responses. If the router signals low-load, the sender increases its sending rate using MI; if the router signals high-load, the sender increases its sending rate using AI; otherwise, if the router signals overload, the sender reduces its sending rate using MD. The core of the VCP protocol is summarized by the following pseudo code.

1) Each router periodically estimates a load factor, and encodes this load factor into the data packets' IP header. This information is then sent back by the receiver to the sender via ACK packets.

2) Based on the load factor it receives, each sender performs one of the following control algorithms:

2.1) For low-load, perform MI;

2.2) For high-load, perform AI;

2.3) For overload, perform MD.

Figure 1 shows the throughput dynamics of two flows sharing one bottleneck link. Clearly, VCP is successful in tracking the bandwidth changes by using MIMD, and achieve fair allocation when the second flow arrives, by using AIMD.
The Internet, however, is much more complex than this simplified example across many dimensions: the link capacities and router buffer sizes are highly heterogeneous, the RTT of flows may differ significantly, and the number of flows is unknown and changes over time. We next describe the details of the VCP protocol, which will be able to handle more realistic environments.

\section{THE VCP PROTOCOL}

In this section, we provide a detailed description of VCP. We begin by presenting three key issues that need to be addressed in the design of VCP. Then, we describe how we address each of these issues in turn.

\subsection{Key Design Issues}

To make VCP a practical approach for the Internet-like environments with significant heterogeneity in link capacities, end-to-end RTTs, router buffer sizes and variable traffic characteristics, we need to address the following three key issues.

Load factor transition point: VCP separates the network load condition into three regions: low-load, high-load and overload. The load factor transition point in VCP represents the boundary between the low-load and high-load regions, which is also the demarcation between applying MI and AI algorithms. The choice of the transition point represents a trade-off between achieving high link utilization and responsiveness to congestion. Achieving high network utilization requires a high value for the transition point. But this choice negatively impacts responsiveness to congestion, which in turn affects the convergence time to achieve fairness. Additionally, given that Internet traffic is inherently bursty [46][56], we require a reliable estimation algorithm of the load factor. We discuss the issue of load factor estimation in Section 3.2.

Setting of congestion control parameters: Using MI for congestion control is often fraught with the danger of instability due to its large variations over short time scales. To maintain stability and avoid large queues at routers, we need to make sure that the aggregate rate of the VCP flows using MI does not overshoot the link capacity. Similarly, to achieve fairness, we need to make sure that a flow enters the AI phase before the link gets congested. In order to satisfy these criteria, we need an appropriate choice of MI, AI and MD parameters that can achieve high utilization while maintaining stability, fairness and small persistent queues. To better understand these issues, we first describe our parameter settings for a simplified network model, where all flows have the same RTT and observe the same state of the network load condition, i.e., all flows obtain the same load factor feedback (Section 3.3). We then generalize our parameter choice for flows with heterogeneous RTTs.

Heterogeneous RTTs: When flows have heterogeneous RTTs, different flows can run different algorithms (i.e., MI, $\mathrm{AI}$, or MD) at a given time. This may lead to unpredictable behavior. The RTT heterogeneity can have a significant impact even when all flows run the same algorithm, if this algorithm is MI. In this case, a flow with a lower RTT can claim much more bandwidth than a flow with a higher RTT. To address this problem, end-hosts need to adjust their MI parameters according to their observed RTTs, as discussed in Section 3.4. 


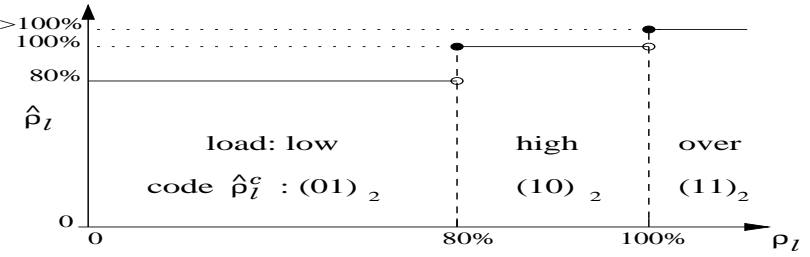

Figure 2: The quantized load factor $\hat{\rho}_{l}$ at a link $l$ is a non-decreasing function of the raw load factor $\rho_{l}$ and can be represented by a two-bit code $\hat{\rho}_{l}^{c}$.

We now discuss these three design issues in greater detail.

\subsection{Load Factor Transition Point}

Consider a simple scenario involving a fixed set of longlived flows. The goal of VCP is to reach a steady state where the system is near full utilization, and the flows use AIMD for congestion control. To achieve this steady state, the choice of the load factor transition point should satisfy three constraints:

- The transition point should be sufficiently high to enable the system to obtain high overall utilization;

- After the flows perform an MD from an overloaded state, the MD step should force the system to always enter the high-load state, not the low-load state;

- If the utilization is marginally lower than the transition point, a single MI step should only lift the system into the high-load state, but not the overload state.

Let $\beta<1$ denote the MD factor, i.e., when using the MD algorithm, the sender reduces the congestion window with the factor $\beta$ (as in Equation (4) in Section 3.3). The first constraint requires a high transition point. This choice coupled with the second condition leads to a high value of $\beta$. However, a very high value of $\beta$ is undesirable as it decreases VCP's response to congestion. For example, if the transition point is $95 \%$, then $\beta>0.95$, and it takes VCP about 14 RTTs to halve the congestion window. At the other end, if we chose $\beta=0.5$ (as in TCP [25]), the transition point can be at most $50 \%$, which reduces the overall network utilization. To balance these conflicting requirements, we chose $\beta=0.875$, the same value used in the DECbit scheme [58]. Given $\beta$, we set the load factor transition point to $80 \%$. This gives us a "safety margin" of $7.5 \%$, which allows the system to operate in the AIMD mode in steady state. In summary, we choose the following three ranges to encode the load factor $\rho_{l}$ (see Figure 2):

- Low-load region: $\hat{\rho}_{l}=80 \%$ when $\rho_{l} \in[0 \%, 80 \%$ );

- High-load region: $\hat{\rho}_{l}=100 \%$ when $\rho_{l} \in[80 \%, 100 \%)$;

- Overload region: $\hat{\rho}_{l}>100 \%$ when $\rho_{l} \in[100 \%, \infty)$.

Thus, the quantized load factor $\hat{\rho}_{l}$ can be represented using a two-bit code $\hat{\rho}_{l}^{c}$, i.e., $\hat{\rho}_{l}^{c}=(01)_{2},(10)_{2}$ and $(11)_{2}$ for $\hat{\rho}_{l}=80 \%, \hat{\rho}_{l}=100 \%$ and $\hat{\rho}_{l}>100 \%$, respectively. The code $(00)_{2}$ is reserved for ECN-unaware source hosts to signal "not-ECN-capable-transport" to ECN-capable routers, which is essential for incremental deployment [57]. The encoded load factor is embedded in the two-bit ECN field in the IP header.
Estimation of the load factor: Due to the bursty nature of the Internet traffic, we need to estimate the load factor over an appropriate time interval, $t_{\rho}$. When choosing $t_{\rho}$ we need to balance two conflicting requirements. On one hand, $t_{\rho}$ should be larger than the RTTs experienced by most flows to factor out the burstiness induced by the flows' responses to congestion. On the other hand, $t_{\rho}$ should be small enough to avoid queue buildup. Internet measurements $[55,30]$ report that roughly $75 \% \sim 90 \%$ of flows have RTTs less than $200 \mathrm{~ms}$. Hence, we set $t_{\rho}=200 \mathrm{~ms}$. During every time interval $t_{\rho}$, each router estimates a load factor $\rho_{l}$ for each of its output links $l$ as [27, 34, 18, 2, 42]:

$$
\rho_{l}=\frac{\lambda_{l}+\kappa_{q} \cdot \widetilde{q}_{l}}{\gamma_{l} \cdot C_{l} \cdot t_{\rho}}
$$

Here, $\lambda_{l}$ is the amount of input traffic during the period $t_{\rho}, \widetilde{q}_{l}$ is the persistent queue length during this period, $\kappa_{q}$ controls how fast the persistent queue drains [18,2] (we set $\kappa_{q}=0.5$ ), $\gamma_{l}$ is the target utilization [42] (set to a value close to 1 ), and $C_{l}$ is the link capacity. The input traffic $\lambda_{l}$ is measured using a packet counter. To measure the persistent queue $\widetilde{q_{l}}$, we use a low-pass filter that samples the instantaneous queue size, $q(t)$, every $t_{q} \ll t_{\rho}$ (we chose $t_{q}=10 \mathrm{~ms}$ ).

\subsection{Congestion Control Parameter Setting}

In this section, we discuss the choice of parameters used by VCP to implement the MI/AI/MD algorithms. To simplify the discussion, we consider a single link shared by flows, whose RTTs are equal to the link load factor estimation period, i.e., $r t t=t_{\rho}$. Hence, the flows have synchronous feedback and their control intervals are also in sync with the link load factor estimation. We will discuss the case of heterogeneous RTTs in Section 3.4.

At any time $t$, a VCP sender performs one of the three actions based on the value of the encoded load factor sent by the network:

$$
\begin{aligned}
& \text { MI : } \quad \operatorname{cwnd}(t+r t t)=\operatorname{cwnd}(t) \times(1+\xi) \\
& \text { AI : } \quad c w n d(t+r t t)=c w n d(t)+\alpha \\
& \text { MD : } \quad c w n d(t+\delta t)=\operatorname{cwnd}(t) \times \beta
\end{aligned}
$$

where $r t t=t_{\rho}, \delta t \rightarrow 0+, \xi>0, \alpha>0$ and $0<\beta<1$. Based on the relationship between the choice of the load factor transition point and the MD parameter $\beta$, we chose $\beta=$ 0.875 (see Section 3.2). We use $\alpha=1.0$ as is in TCP [25].

Setting the MI parameter: The stability of VCP is dictated by the MI parameter $\xi$. In network-based rate allocation approaches like $\mathrm{XCP}$, the rate increase of a flow at any time is proportional to the spare capacity available in the network [35]. Translating this into the VCP context, we require the $\mathrm{MI}$ of the congestion window to be proportional to $1-\hat{\rho}_{l}$ where $\hat{\rho}_{l}$ represents the current load factor. During the MI phase, the current sending rate of each flow is proportional to the current load factor $\hat{\rho}_{l}$. Consequently, we obtain

$$
\xi(\hat{\rho})=\kappa \cdot \frac{1-\hat{\rho}_{l}}{\hat{\rho}_{l}},
$$

where $\kappa$ is a constant that determines the stability of VCP and controls the speed to converge toward full utilization. Based on analyzing the stability properties of this algorithm (see Theorem 1 in Section 5), we set $\kappa=0.25$. Since end- 
hosts only obtain feedback on the utilization region as opposed to the exact value of the load factor, they need to make a conservative assumption that the network load is near the transition point. Thus, the end-hosts use the value of $\xi(80 \%)=0.0625$ in the MI phase.

\subsection{Handling RTT Heterogeneity with Parameter Scaling}

Until now, we have considered the case where competing flows have the same RTT, and this RTT is also equal to the load factor estimation interval, $t_{\rho}$. In this section, we relax these assumptions by considering flows with heterogeneous RTTs. To offset the impact of the RTT heterogeneity, we need to scale the congestion control parameters used by the end-hosts according to their RTTs.

Scaling the MI/AI parameters: Consider a flow with a round trip time $r t t$, and assume that all the routers use the same interval, $t_{\rho}$, to estimate the load factor on each link. Let $\xi$ and $\alpha$ represent the unscaled MI and AI parameters as described in Section 3.3, where all flows have an identical RTT $\left(=t_{\rho}\right)$. To handle the case of flows with different RTTs, we set the scaled MI/AI parameters $\xi_{s}$ and $\alpha_{s}$ as follows: ${ }^{1}$

$$
\begin{array}{ll}
\text { For MI }: & \xi_{s} \leftarrow(1+\xi)^{\frac{r t t}{t_{\rho}}}-1, \\
\text { For AI }: & \alpha_{s} \leftarrow \alpha \cdot \frac{r t t}{t_{\rho}} .
\end{array}
$$

An end-host uses the scaled parameters $\xi_{s}$ and $\alpha_{s}$ in (2) and (3) to adjust the congestion window after each RTT. The scaling of these parameters emulates the behavior of all flows having an identical RTT, which is equal to $t_{\rho}$. The net result is that over any time period, the window increase under either MI or AI is independent of the flows' RTTs. Thus, unlike TCP, VCP flow's throughput is not affected by the RTT heterogeneity [44, 53, 16].

Handling MD: MD is an impulse-like operation that is not affected by the length of the RTT. Hence, the value of $\beta$ in (4) needs not to be scaled with the RTT of the flow. However, to avoid over reaction to the congestion signal, a flow should perform an MD at most once during an estimation interval $t_{\rho}$. Upon getting the first load factor feedback that signals congestion (i.e., $\hat{\rho}_{l}^{c}=(11)_{2}$ ), the sender immediately reduces its congestion window cwnd using MD, and then freezes the cwnd for a time period of $t_{\rho}$. After this period, the end-host runs AI for one RTT in order to obtain the new load factor.

Scaling for fair rate allocation: RTT-based parameter scaling, as described above, only ensures that the congestion windows of two flows with different RTTs converge to the same value in steady state. However, this does not guarantee fairness as the rate of the flow is still inversely proportional to its RTT, i.e., rate $=$ cwnd $/ r t t$. To achieve fair rate allocation, we need to add an additional scaling factor to the AI algorithm. To illustrate why this is the case, consider the simple AIMD control mechanism applied to two competing flows where each flow $i(=1,2)$ uses a separate AI parameter $\alpha_{i}$ but a common MD parameter $\beta$. At the end of the $M$-th

\footnotetext{
${ }^{1}$ Equation (6) is the solution for $1+\xi=\left(1+\xi_{s}\right)^{\frac{t \rho}{r t t}}$ where the right-hand side is the MI amount of a flow with the RTT value $r t t$, during a time interval $t_{\rho}$. Similarly, Equation (7) is obtained by solving $1+\alpha=1+\frac{t_{\rho}}{r t t} \alpha_{s}$.
}

Table 1: VCP Parameter Setting

\begin{tabular}{||c|c|l||}
\hline \hline Para & Value & \multicolumn{1}{|c}{ Meaning } \\
\hline \hline$t_{\rho}$ & $200 \mathrm{~ms}$ & the link load factor measurement interval \\
$t_{q}$ & $10 \mathrm{~ms}$ & the link queue sampling interval \\
$\gamma_{l}$ & 0.98 & the link target utilization \\
$\kappa_{q}$ & 0.5 & how fast to drain the link steady queue \\
\hline$\kappa$ & 0.25 & how fast to probe the available bw (MI) \\
$\alpha$ & 1.0 & the AI parameter \\
$\beta$ & 0.875 & the MD parameter \\
\hline \hline
\end{tabular}

congestion epoch that includes $n>1$ rounds of AI and one round of MD in each epoch, we have

$$
\operatorname{cwnd}_{i}(M)=\beta \cdot\left[\operatorname{cwnd}_{i}(M-1)+n \cdot \alpha_{i}\right] .
$$

Eventually, each flow $i$ achieves a congestion window that is proportional to its AI parameter, $\alpha_{i}$. Indeed, the ratio of the congestion windows of the two flows approaches $\alpha_{1} / \alpha_{2}$ for large values of $M$, and $n>1$ :

$$
\begin{aligned}
\frac{\operatorname{cwnd}_{1}(M)}{\operatorname{cwnd}_{2}(M)} & =\frac{\operatorname{cwnd}_{1}(M-1) / n+\alpha_{1}}{\operatorname{cwnd}_{2}(M-1) / n+\alpha_{2}} \\
& =\frac{\operatorname{cwnd}_{1}(M-2) / n^{2}+\alpha_{1} / n+\alpha_{1}}{\operatorname{cwnd}_{2}(M-2) / n^{2}+\alpha_{1} / n+\alpha_{2}} \\
& =\cdots \rightarrow \frac{\alpha_{1}}{\alpha_{2}} .
\end{aligned}
$$

Hence, to allocate bandwidth fairly among two flows, we need to scale each flow's AI parameter $\alpha_{i}$ using its own RTT. For this purpose, we use $t_{\rho}$ as a common-base RTT for all the flows. Thus, the new AI scaling parameter, $\alpha_{\text {rate }}$, becomes

$$
\text { For AI : } \quad \alpha_{\text {rate }} \leftarrow \alpha_{s} \cdot \frac{r t t}{t_{\rho}}=\alpha \cdot\left(\frac{r t t}{t_{\rho}}\right)^{2} \text {. }
$$

\subsection{Summary of Parameters}

Table 1 summarizes the set of VCP router/end-host parameters and their typical values. We note that throughout all the simulations reported in this paper, we use the same parameter values. This suggests that VCP is robust in a large variety of environments.

\section{PERFORMANCE EVALUATION}

In this section, we use extensive ns2 simulations to evaluate the performance of VCP for a wide range of network scenarios [19] including varying the link capacities in the range [100Kbps, $5 \mathrm{Gbps}]$, round trip times in the range [1ms, $1.5 \mathrm{~s}]$, numbers of long-lived, FTP-like flows in the range [1, 1000], and arrival rates of short-lived, web-like flows in the range $\left[1 s^{-1}, 1000 s^{-1}\right]$. We always use two-way traffic with congestion resulted in the reverse path. The bottleneck buffer size is set to the bandwidth-delay product, or two packets per flow, whichever is larger. The data packet size is 1000 bytes, while the ACK packet is 40 bytes. All simulations are run for at least 120 s to ensure that the system has reached its steady state. The average utilization statistics neglect the first $20 \%$ of simulation time. For all the time-series graphs, utilization and throughput are averaged over $500 \mathrm{~ms}$ interval, while queue length and congestion window are sampled every $10 \mathrm{~ms}$. We use a fixed set of VCP parameters listed in Table 1 for all the simulations in this paper. 

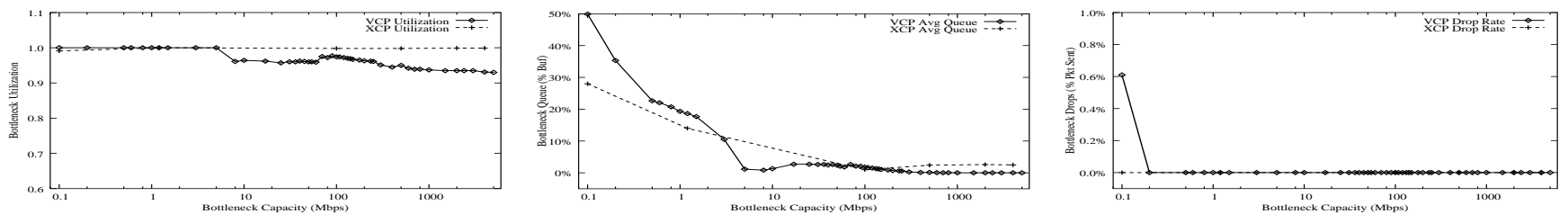

Figure 3: VCP with the bottleneck capacity ranging from 100Kbps to 5Gbps. It achieves high utilization and almost no packet loss with decreasing bottleneck queue as the capacity increases. Note the logarithmic scale of the $x$-axis in this figure and the next one.
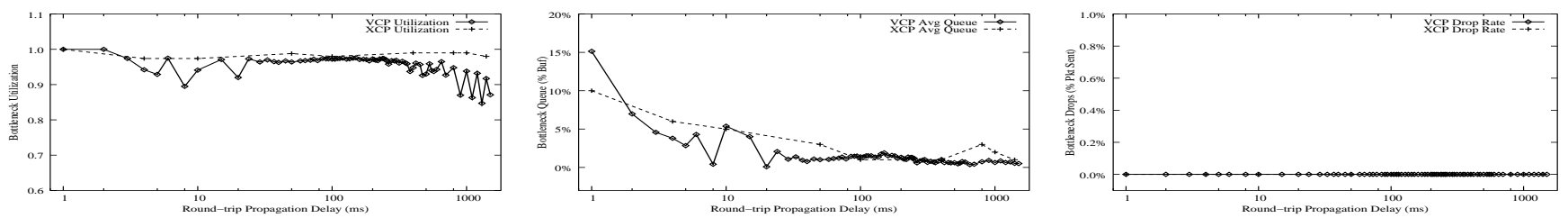

Figure 4: VCP with the round-trip propagation delay ranging from $1 \mathrm{~ms}$ to $1500 \mathrm{~ms}$. It is able to achieve reasonably high utilization, low persistent queue and no packet loss.
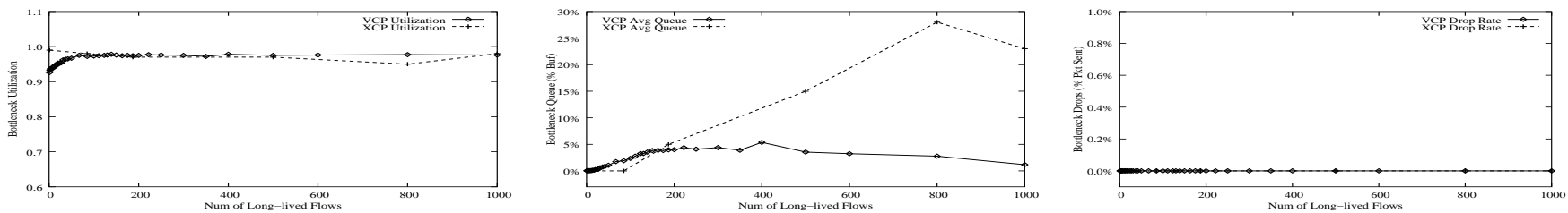

Figure 5: VCP with the number of long-lived, FTP-like flows ranging from 1 to 1000. It achieves high utilization with more bursty bottleneck queue for higher number of FTP flows.

These simulation results demonstrate that, for a wide range of scenarios, VCP is able to achieve exponential convergence to high utilization, low persistent queue, negligible packet drop rate and reasonable fairness, except its significantly slower fairness convergence speed compared to XCP.

\subsection{One Bottleneck}

We first evaluate the performance of VCP for the simple case of a single bottleneck link shared by multiple VCP flows. We study the effect of varying the link capacity, the round-trip times, the number of flows on the performance of VCP. The basic setting is a $150 \mathrm{Mbps}$ link with $80 \mathrm{~ms}$ RTT where the forward and reverse path each has 50 FTP flows. We evaluate the impact of each network parameter in isolation while retaining the others as the basic setting.

Impact of Bottleneck Capacity: As illustrated in Figure 3, we observe that VCP achieves high utilization $(\geq 93 \%)$ across a wide range of bottleneck link capacities varying from $100 \mathrm{Kbps}$ to $5 \mathrm{Gbps}$. The utilization gap in comparison to $\mathrm{XCP}$ is at most $7 \%$ across the entire bandwidth range. Additionally, as we scale the bandwidth of the link, the average (maximal) queue length decreases to about $0.01 \%$ (1\%) buffer size. The absolute persistent queue length is very small for higher capacities, leading to negligible packet drop rates (zero packet drops for many cases). At extremely low capacities, e.g., 100Kbps (per-flow BDP of 0.02 packets), the bottleneck average queue significantly increases to $50 \%$ of the buffer size, resulting in roughly $0.6 \%$ packet loss. This happens because the AI parameter setting $(\alpha=1.0)$ is too large for such low capacities.
Impact of Feedback Delay: We fix the bottleneck capacity at $150 \mathrm{Mbps}$ and vary the round-trip propagation delay from $1 \mathrm{~ms}$ to $1500 \mathrm{~ms}$. As shown in Figure 4, we notice that in most cases, the bottleneck utilization is higher than $90 \%$, and the average (maximal) queue is less than $5 \%$ $(15 \%)$ of the buffer size. We also observe that the RTT parameter scaling is sensitive to very low values of RTT (e.g., 1ms), thereby causing the average (maximal) queue length to grow to about $15 \%(45 \%)$ of the buffer size. For the RTT values larger than $800 \mathrm{~ms}$, VCP obtains lower utilization ( $85 \% \sim 94 \%)$ since the link load factor measurement interval $t_{\rho}=200 \mathrm{~ms}$ is much less than the RTTs of the flows. As a result, the load condition measured in each $t_{\rho}$ shows variations due to the bursty nature of window-based control. This can be compensated by increasing $t_{\rho}$; but the trade-off is that the link load measurement will be less responsive causing the queue length to grow. In all these cases, we did not observe any packet drops in VCP.

Impact of Number of Long-lived Flows: With an increase in the number of forward FTP flows, we notice that the traffic gets more bursty, as shown by the increasing trend of the bottleneck maximal queue. However, even when the network is very heavily multiplexed by 1000 flows (i.e., the average per-flow BDP equals to only 1.5 packets), the maximal queue is still less than $38 \%$ of the buffer size. The average queue is consistently less than $5 \%$ buffer size as shown in Figure 5 across all these cases. For the heavily multiplexed cases, VCP even slightly outperforms XCP.

Impact of Short-lived Traffic: To study VCP's performance in the presence of variability and burstiness in flow 

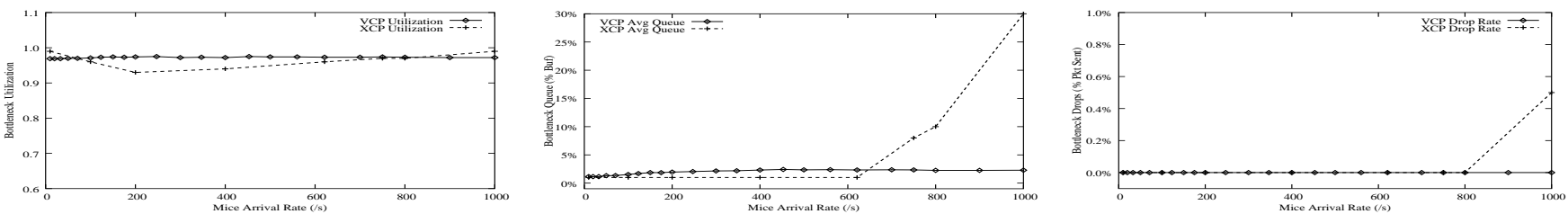

Figure 6: Similar to XCP, VCP remains efficient with low persistent queue and zero packet loss given the short-lived, web-like flows arriving/departing at a rate from $1 / \mathrm{s}$ to $1000 / \mathrm{s}$.
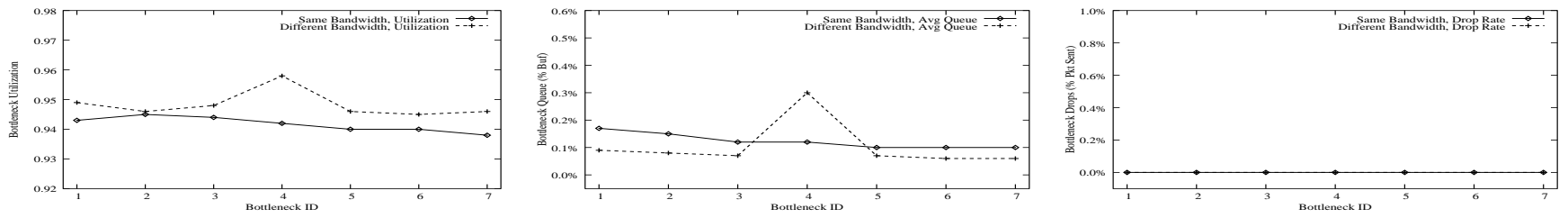

Figure 7: VCP with multiple congested bottlenecks. For either all the links have the same capacity (100Mbps), or the middle link \#4 has lower capacity (50Mbps) than the others, VCP consistently achieves high utilization, low persistent queue and zero packet drop on all the bottlenecks.
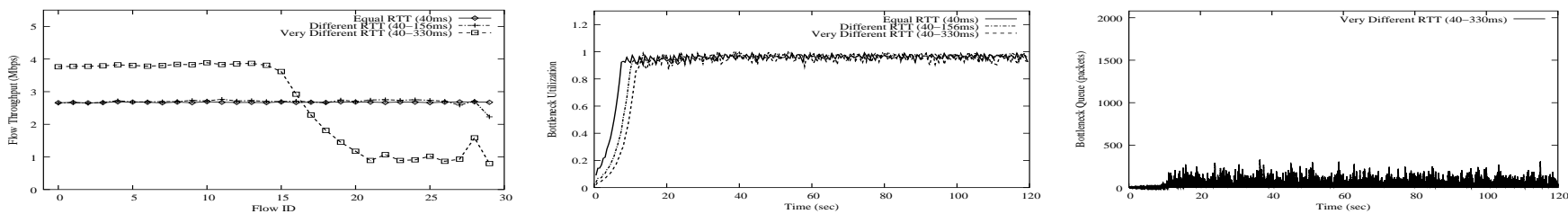

Figure 8: To some extent, VCP distributes bandwidth fairly among competing flows with either equal or different RTTs. In all the case, it maintains high utilization, keeps small queue and drops no packet.

arrivals, we add web traffic into the network. These flows arrive according to the Poisson process, with the average arrival rate varying from $1 / \mathrm{s}$ to $1000 / \mathrm{s}$. Their transfer size obeys the Pareto distribution with an average of 30 packets. This setting is consistent with the real-world web traffic model [11]. As shown by Figure 6, the bottleneck always maintains high utilization with small queue lengths and zero packet drops, similar to XCP.

In summary, we note that across a wide range of network configurations with a single bottleneck link, VCP can achieve comparable performance as XCP including high utilization, low persistent queues, and negligible packet drops. All these results are achieved with a fixed set of parameters shown in Table 1.

\subsection{Multiple Bottlenecks}

Next, we study the performance of VCP with a more complex topology of multiple bottlenecks. For this purpose, we use a typical parking-lot topology with seven links. All the links have a $20 \mathrm{~ms}$ one-way propagation delay. There are 50 FTP flows traversing all the links in the forward direction, and 50 FTP flows in the reverse direction as well. In addition, each individual link has 5 cross FTP flows traversing in the forward direction. We run two simulations. First, all the links have 100Mbps capacity. Second, the middle link \#4 has the smallest capacity of only $50 \mathrm{Mbps}$, while all the others have the same capacity of $100 \mathrm{Mbps}$.

Figure 7 shows that for both cases, VCP performs as good as in the single-bottleneck scenarios. For the first case, VCP achieves $94 \%$ average utilization, less than $0.2 \%$-buffer-size average queue length and zero packet drops at all the bottlenecks. When we lower the capacity of the middle link, its average utilization increases slightly to $96 \%$, with the largest maximal queue representing only $6.4 \%$ buffer size. In comparison to XCP, one key difference is that VCP penalizes long flows more than short flows. For example, in the second case, VCP allocates $0.39 \mathrm{Mbps}$ to each long flow, and $4.96 \mathrm{Mbps}$ to each cross flow that passes the middle link; while all these flows get about $0.85 \mathrm{Mbps}$ under XCP. We discuss the reason behind this in Section 5 .

\subsection{Fairness}

TCP flows with different RTTs achieve bandwidth allocation that is proportional to $1 / r t t^{z}$ where $1 \leq z \leq 2$ [44]. VCP alleviates this issue to some extent. Here we look at the RTT-fairness of VCP. We have 30 FTP flows sharing a single $90 \mathrm{Mbps}$ bottleneck, with $30 \mathrm{FTP}$ flows on the reverse path. We perform three sets of simulations: (a) the same RTT; (b) small RTT difference; (c) huge RTT difference. We will see that VCP is able to allocate bottleneck bandwidth fairly among competing flows, as long as their RTTs are not significantly different. This capability degrades as the RTT heterogeneity increases.

In the case where all the flows have a common RTT or have a small RTT difference, VCP achieves a near-even distribution of the capacity among the competing flows (refer to Figure 8). However, when the flows have significantly different RTTs, VCP does not distribute the bandwidth fairly between the flows that have huge RTT variation (with throughput ratio of up to 5). This fairness discrepancy occurs due to 

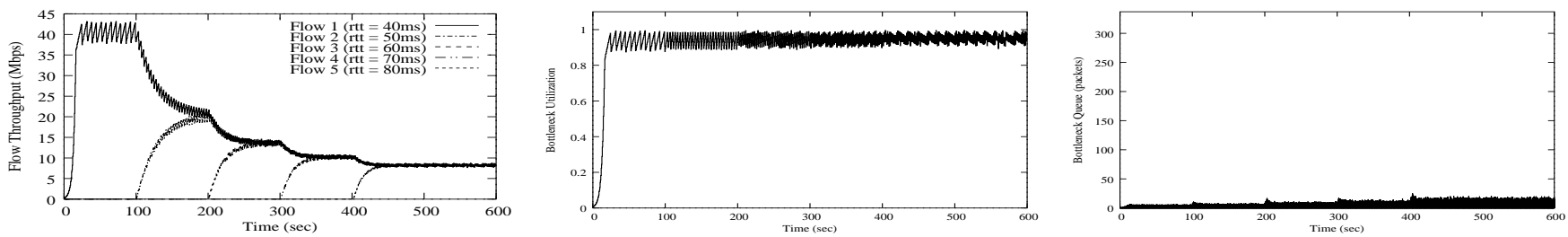

Figure 9: VCP converges onto good fairness, high utilization and small queue. However, its fairness convergence takes significantly longer time than XCP.
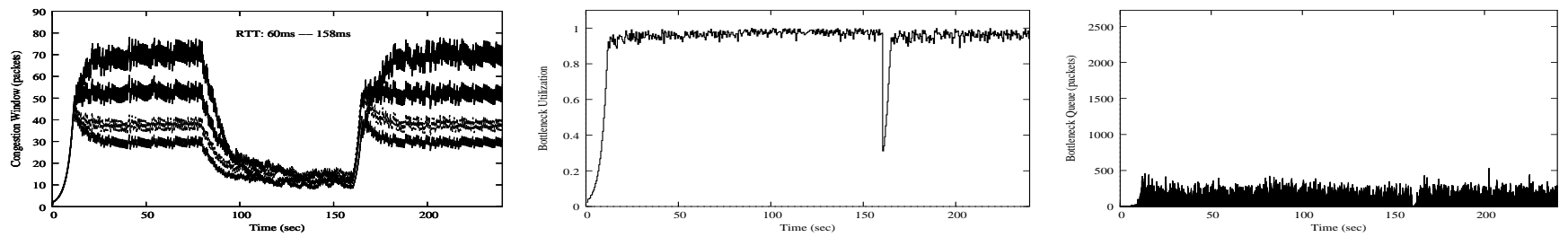

Figure 10: VCP is robust against and responsive to sudden, considerable traffic demand changes, and at the same time maintains low persistent bottleneck queue.

the following reason. A flow with a very high RTT is bound to have high values for their MI and AI parameters due to parameter scaling (see Section 3.4). Due to practical operability constraints, we place artificial bounds on the actual values of these parameters (specifically the MI parameter) to prevent sudden bursts from VCP flows which can cause the persistent queue length at the bottleneck link to increase substantially. These bounds restrict the throughput of flows with very high RTTs.

\subsection{Dynamics}

All the previous simulations focus on the steady-state behavior of VCP. Now, we investigate its short-term dynamics.

Convergence Behavior: To study the convergence behavior of VCP, we revert to the single bottleneck link with a bandwidth of $45 \mathrm{Mbps}$ where we introduce 5 flows into the system, one after another, with starting times separated by $100 \mathrm{~s}$. We also set the RTT values of the five flows to different values. The reverse path has 5 flows that are always active. Figure 9 illustrates that VCP reallocates bandwidth to new flows whenever they come in without affecting its high utilization or causing large instantaneous queue. (All the figures of queue dynamics in this paper use the router buffer size to scale their queue-length axis.) However, VCP takes a much longer time than XCP to converge to the fair allocation. We theoretically quantify the fairness convergence speed for VCP in Theorem 4 in Section 5.

Sudden Demand Change: We illustrate how VCP reacts to sudden changes in traffic demand using a simple simulation. Consider an initial setting of 50 forward FTP flows with varying RTTs (uniformly chosen in the range $[50 \mathrm{~ms}$, $150 \mathrm{~ms}]$ ) sharing a $200 \mathrm{Mbps}$ bottleneck link. There are 50 FTP flows on the reverse path. At $t=80 \mathrm{~s}, 150$ new forward FTP flows become active; then they leave at 160s. Figure 10 clearly shows that VCP can adapt sudden fluctuations in the traffic demand. (The left figure draws the congestion window dynamics for four randomly chosen flows.) When the new flows enter the system, the flows adjust their rates to the new fair share while maintaining the link at high utilization. At $\mathrm{t}=160 \mathrm{~s}$, when three-fourths of the flows depart creating a sudden drop in the utilization, the system quickly discovers this and ramps up to $95 \%$ utilization in about 5 seconds. Notice that during the adjustment period, the bottleneck queue remains much lower than its full size. This simulation shows that VCP is responsive to sudden, significant decreases/increases in the available bandwidth. This is no surprise because VCP switches to the MI mode which by nature can track any bandwidth change in logarithmic time (see Theorem 3 in Section 5).

We have also performed a variety of other simulations to show VCP's ability to provide bandwidth differentiation. Due to the limited space we are unable to present the results here. We refer the reader to our technical report for more details [66].

\section{A FLUID MODEL}

To obtain insight into the behavior of $\mathrm{VCP}$, in this section, we consider a simple fluid model, and analyze its stability and fairness properties. We also analyze VCP's efficiency and fairness convergence speed.

Our model approximates the behavior of VCP using a load-factor guided algorithm which combines the MI and AI steps of VCP as described in (2) and (3) in Section 3.3:

$$
\dot{w}_{i}(t)=\frac{1}{T} \cdot\left[w_{i}(t) \cdot \xi(\rho(t))+\alpha\right]
$$

with the MI parameter

$$
\xi(\rho(t))=\kappa \cdot \frac{1-\rho(t)}{\rho(t)},
$$

where $\kappa>0$ is the stability coefficient of the MI parameter. In the remainder of this section we will refer to this model as the MIAIMD model. It assumes infinite router buffers, and that end-hosts know the exact value of the load factor $\rho(t)$, as computed by the routers.

We start our analysis by considering a single bottleneck link traversed by $N$ flows that have the same RTT, $T$. As shown in Figure 11, the load factor $\rho(t)$ received by the source at a time $t$ is computed based on the sender's rate at time $t-T$, 


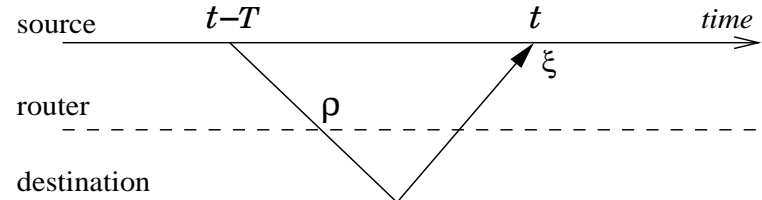

Figure 11: A simplified VCP model. The source sending rate at time $t-T$ is used by the router to calculate a load factor $\rho$, which is echoed back from the destination to the source at time $t$. Then the source adjusts its MI parameter $\xi(\rho(t))$ based on the load factor $\rho(t)$.

$$
\rho(t)=\frac{\sum_{i=1}^{N} w_{i}(t-T)}{\gamma C T}
$$

where $w_{i}(t)$ is the flow $i$ 's congestion window at time $t, C$ is the link capacity, and $0<\gamma \leq 1$ is the target link utilization. We assume that $w_{i}(t)$ is a positive, continuous and differentiable function, and $T$ is a constant.

Since $\xi(\rho(t))$ is proportional to the available bandwidth, the MIAIMD algorithm tracks the available bandwidth exponentially fast and thus achieves efficiency. It also converges to fairness as we will show in Theorem $2 .^{2}$

Using (9) to sum over all $N$ flows yields

$$
\dot{w}(t)=\frac{1}{T} \cdot[w(t) \cdot \xi(\rho(t))+N \alpha]
$$

where $w(t)=\sum_{i=1}^{N} w_{i}(t)$ is the sum of all the congestion windows. This result, together with (10) and (11), leads to

$$
\dot{w}(t)=\frac{1}{T} \cdot\left\{\kappa \cdot w(t) \cdot\left[\frac{\gamma C T}{w(t-T)}-1\right]+N \alpha\right\}
$$

where $w(t)>0$. We assume the initial condition $w(t)=N$ (i.e., $\left.w_{i}(t)=1\right)$, for all $t \in[-T, 0]$. In [66], we prove the following global stability result.

Theorem 1. Under the model (9), (10) and (11) where a single bottleneck is shared by a set of synchronous flows with the same RTT, if $\kappa \leq \frac{1}{2}$, then the delayed differential equation (13) is globally asymptotically stable with a unique equilibrium $w^{*}=\gamma C T+N \frac{\alpha}{\kappa}$, and all the flows have the same steady-state rate $r_{i}^{*}=\frac{\gamma C}{N}+\frac{\alpha}{\kappa T}$.

This result has two implications. First, the sufficient condition $\kappa \leq \frac{1}{2}$ holds for any link capacity, any feedback delay, and any number of flows. Furthermore, the global stability result does not depend on the network parameters. Second, this result is optimal in that at the equilibrium, the system achieves all the design goals: high utilization, fairness, zero steady-state queue length, and zero packet loss rate - this is because we can always adjust $\gamma$ such that the system stabilizes at a steady-state utilization slightly less than 1.

Importance of $\gamma$ : While (11) defines $\gamma$ as the target utilization, the actual utilization is $\frac{w^{*}}{C T}=\gamma+\frac{\alpha}{\kappa P}$ where $P=\frac{C T}{N}$ is the per-flow BDP. To achieve a certain target utilization $\gamma^{*}, \gamma$ should be treated as a control variable and set to $\gamma=\gamma^{*}-\frac{\alpha}{\kappa P}$. For more details on how to make this adjustment process automatic without even knowing $\alpha, \kappa$ and $P$, we refer the reader to [66].

\footnotetext{
${ }^{2}$ Theorem 2 actually proves the max-min fairness for a general multiple-bottleneck topology. For a single link, max-min fairness means each flow gets an equal share of the link capacity.
}

Next, we consider a more general multiple-bottleneck network topology. Let $\rho^{i}(t)$ denote the maximal link load factor on flow $i$ 's path $L_{i}$ that includes a subset of links, i.e., $L_{i}=\{l \mid$ flow $i$ traverses link $l\}$. The MI parameter of flow $i$ is then

$$
\xi\left(\rho^{i}(t)\right)=\kappa \cdot\left[\frac{1}{\rho^{i}(t)}-1\right]
$$

where $\rho^{i}(t)=\max _{l \in L_{i}} \rho_{l}(t), \rho_{l}(t)=\frac{\sum_{i \in I_{l}} w_{i}(t-T)}{\gamma C_{l} T}$, and the subset of flows $I_{l}=\{i \mid$ flow $i$ traverses link $l\}$. We prove the following fairness result in [66].

Theorem 2. In a multiple-bottleneck topology where all flows have the same round-trip time $T$, if there exists a unique equilibrium, then the algorithm defined by (9) and (14) allocates a set of max-min fair rates $r_{i}^{*}=\frac{\alpha}{\kappa T\left(1-\frac{1}{\max _{l \in L_{i}} \rho_{l}^{*}}\right)}$
$\sum_{i \in I_{l}} w_{i}^{*}$ where $\rho_{l}^{*}=\frac{\sum_{i \in I_{l}} w_{i}^{*}}{\gamma C T}$.

To better understand this result note that a flow's sending rate is determined by the most congested bottleneck link on its path. Thus, the flows traversing the most congested bottleneck links in the system will naturally experience the lowest throughputs.

Having established the stability and fairness properties of the MIAIMD model, we now turn our attention on the convergence of the VCP protocol. The following two theorems, proved in [66], give the convergence properties.

Theorem 3. The VCP protocol takes $O(\log C)$ RTTs to claim (or release) a major part of any spare (or over-used) capacity $C$.

Theorem 4. The VCP protocol takes $O(P \log \Delta P) R T T$ s to converge onto fairness for any link, where $P$ is the perflow bandwidth-delay product, and $\Delta P>1$ is the largest congestion window difference between flows sharing that link.

Not surprisingly, due to the use of MI in the low-load region, VCP converges exponentially fast to high utilization. On the other hand, VCP's convergence time to fairness is similar to other AIMD-based protocols, such as TCP+AQM. In contrast, explicit feedback schemes like XCP require only $O(\log \Delta P)$ RTTs to converge to fairness. This is because the end-host based AIMD algorithms improve fairness per AIMD epoch, which includes $O(P)$ rounds of AI and one round of $\mathrm{MD}$, while the equivalent operation in XCP takes only one RTT.

The VCP protocol can be viewed as an approximation of the MIAIMD model along three axes. First, the MIAIMD model uses the exact load factor feedback, $\rho(t)$, while VCP uses a quantized value of the load factor. Second, in the $\mathrm{MI}$ and AI phases, VCP uses either the multiplicative factor or the additive factor term, but not both as the MIAIMD model does. Third, in the overload region, VCP applies a constant MD parameter $\beta$ instead of $\xi(\rho(t))$.

The comparison between the simulation results of VCP and the analytical results of the MIAIMD model suggests that the two differ most notably in terms of the fairness model. While in the case of multiple bottleneck links, the MIAIMD model achieves max-min fairness [4], VCP tends to allocate more bandwidth to flows that traverse fewer bottleneck links (see Section 4.2). This is because VCP relies on the quantized representation of the load factor instead of the exact value. 


\section{DISCUSSIONS}

Since VCP switches between MI, AI, and MD algorithms based on the load factor feedback, there are natural concerns with respect to the impact of these switches on the system stability, efficiency, and fairness, particularly in systems with highly heterogeneous RTTs. We discuss these concerns in this section. We discuss VCP's TCP-friendliness and incremental deployment in [66].

\subsection{Stability under Heterogeneous Delays}

Although the MIAIMD model presented in Section 5 is provably stable, it assumes synchronous feedback. To accommodate heterogeneous delays, VCP scales the MI/AI parameters such that flows with different RTTs act as if they were having the same RTT. This scaling mechanism is also essential to achieving fair bandwidth allocation, as discussed in Section 3.4.

In normal circumstances, VCP makes a transition to MD only from AI. However, even if VCP switches directly from $\mathrm{MD}$ to $\mathrm{MI}$, if the demand traffic at the router does not change significantly, VCP will eventually slide back into AI.

Finally, to prevent the system from oscillating between MI and MD, we set the load factor transition point $\hat{\rho}_{l}$ to $80 \%$, and set the MD parameter $\beta$ to $0.875>\hat{\rho}_{l}$. This gives us a safety margin of $7.5 \%$.

The extensive simulation results presented in Section 4 suggest that VCP is indeed stable over a large variety of network scenarios including per-flow bandwidths from $2 \mathrm{Kbps}$ to $100 \mathrm{Mbps}$ and RTTs from $1 \mathrm{~ms}$ to $1.5 \mathrm{~s}$.

\subsection{Influences of Mode Sliding}

From an efficiency perspective, VCP's goal is to bring and maintain the system into the high utilization region. While MI enables VCP to quickly reach the high link utilization, VCP needs also to make sure that the system remains in this state. The main mechanisms employed by VCP to achieve this goal is the scaling of the MI/AI parameters for flows with different RTTs. In addition to improving fairness, this scaling is essential to avoid oscillations. Otherwise, a flow with a low RTT may apply MI several times during the estimation interval, $t_{\rho}$, of the link load factor. Other mechanisms employed by VCP to maintain high efficiency include choosing an appropriate value of the MD parameter to remain in the high utilization region, using a safety margin between MI and AI, and bounding the burstiness (Section 4.3).

As discussed in Section 3.4, there are two major concerns with respect to fairness. First, a flow with a small RTT probes the network faster than a flow with a large RTT. Thus, the former may increase its bandwidth much faster than the latter. Second, it will take longer for a large-RTT flow to switch from MI to AI than a small-RTT flow. This may give the large-RTT flow an unfair advantage. VCP addresses the first issue by using the RTT scaling mechanism (see (6)-(7)). To address the second issue, VCP bounds the MI gain, as discussed in Section 4.3. To illustrate the effectiveness of limiting the MI gain, Figure 12 shows the congestion window evolution for two flows with RTTs of $50 \mathrm{~ms}$ and $500 \mathrm{~ms}$, respectively, traversing a single $10 \mathrm{Mbps}$ link. At time $12.06 \mathrm{~s}$, the $50 \mathrm{~ms}-\mathrm{RTT}$ flow switches from MI to AI. In contrast, due to its larger RTT, the 500ms-RTT flow keeps performing MI until time $12.37 \mathrm{~s}$. However, because VCP limits the MI gain of the 500ms-RTT flow, the additional

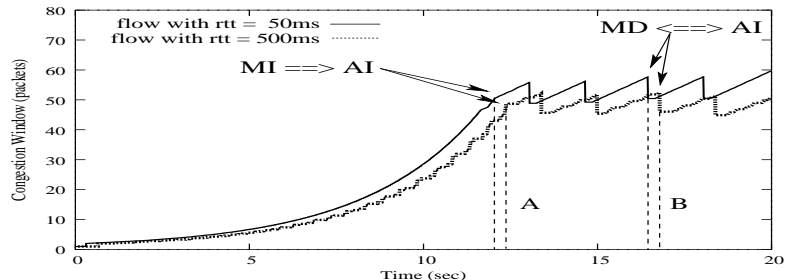

Figure 12: The congestion window dynamics of two flows with dramatically different RTTs $(50 \mathrm{~ms}$ vs. $500 \mathrm{~ms}$ ). Due to its longer delay, the larger-RTT flow always slides its mode later than the one with smaller RTT (see the regions labeled as $\mathrm{A}$ and $\mathrm{B}$ ). However, the effect of this asynchronous switching is accommodated by VCP and does not prevent it from maintaining stability and achieving efficiency and fairness.

bandwidth acquired by this flow during the 0.31 s interval is only marginal when compared to the bandwidth acquired by the $50 \mathrm{~ms}-\mathrm{RTT}$ flow.

\section{RELATED WORK}

This paper builds upon a great body of related work, particularly XCP [35], TCP [25, 1, 17, 51], AIMD [10, 29], $\operatorname{AQM}[18,2,42]$ and $\operatorname{ECN}[57,58]$. Congestion control is pioneered by TCP and AIMD. The research on AQM starts from RED [18, 47], followed by Blue [14], REM [2], PI controller [23], AVQ [21, 42], and CHOKe [54], etc. Below we relate VCP to three categories of congestion control schemes and a set of analytical results.

Explicit rate based schemes: $\mathrm{XCP}$ regulates source sending rate with decoupled efficiency control and fairness control and achieves excellent performance. ATM ABR service (e.g., see $[40,9,33,27,34])$ previously proposes explicit rate control. VCP learns from these schemes. In contrast, VCP is primarily an end-host based protocol. This key difference brings new design challenges not faced by XCP (and the ATM ABR schemes) and thus VCP is not just a "twobit" version of XCP. The idea of classifying network load into different regions is originally presented in [28]. The link load factor is suggested as a congestion signal in [27], based on which VCP quantizes and encodes it for a more compact representation for the degree of congestion. MaxNet [65] uses the maximal congestion information among all the bottlenecks to achieve max-min fairness. QuickStart [26] occasionally uses several bits per packet to quickly ramp up source sending rates. VCP is complementary to QuickStart as it constantly uses two bits per packet.

Congestion notification based schemes: For high BDP networks, according to [35], the performance gap between $\mathrm{XCP}$ and TCP+RED/REM/AVQ/CSFQ [60] with one-bit ECN support seems large. VCP generalizes one-bit ECN and applies some ideas from these AQM schemes. For example, RED's queue-averaging idea, REM's match-rate-clearbuffer idea and AVQ's virtual-capacity idea obviously find themselves in VCP's load factor calculation in Equation (1). This paper demonstrates that the marginal performance gain from one-bit to two-bit ECN feedback could be significant. On the end-host side, two-bit ECN is also used to choose different decrease parameters for TCP in [13], which is very different from the way VCP uses. GAIMD [68] and the bino- 
mial control [3] generalize the AIMD algorithm, while VCP goes even further to combine MIMD with AIMD.

Pure end-to-end schemes: Recently there have been many studies on the end-to-end congestion control for high BDP networks. HighSpeed TCP [15] extends the standard TCP by adaptively setting the increase/decrease parameters according to the congestion window size. H-TCP [45] employs an adaptive AIMD with its parameters set as functions of the elapsed time since the last congestion event. Adaptive TCP [38] also applies dynamic AIMD parameters with respect to the changing network conditions. STCP [36] changes to a fixed MIMD algorithm. FAST [31] uses queueing delay, like TCP Vegas [6], instead of packet loss, as its primary congestion signal and improves Vegas' AdditiveIncrease-Additive-Decrease policy with a proportional controller. BIC [67, 59] adds a binary search phase into the standard TCP to probe the available bandwidth in a logarithmic manner. LTCP [5] layers congestion control of two scales for high speed, large RTT networks. TCP Westwood [8] enhances the loss-based congestion detector using more robust bandwidth estimation techniques. All these end-to-end schemes do not need explicit feedback. Therefore, it is hard for them to achieve both low persistent bottleneck queue length and almost zero congestion-caused packet loss rate. VCP does need explicit two-bit ECN but is able to maintain low queue and almost zero loss. However, it is unclear whether these end-to-end schemes, if given AQM/ECN support from network, can achieve similar performance as VCP in high BDP networks.

Analytical Results: The nonlinear optimization framework $[37,48,41]$ provides the above schemes a unified theoretic underpin and proposes a class of control algorithms. The local stability of the algorithms when homogeneous delay is present is considered by $[32,62]$ and then extended to the case of heterogeneous delays by [50]. The local stability of a modified algorithm for the case of heterogeneous delays is proved by [70], which establishes a model that is similar to what we show in Section 5. In contrast, a global stability result is obtained in this paper for the case of a single bottleneck with homogeneous delays. The global stability of more general congestion controllers are considered by other researchers, e.g., in [63, 12, 69].

Variable-structure control with sliding modes has a long history in control theory [61]. It is useful when a set of features are desired in a system but no single algorithm can provide all of them. In computer networking areas, it has been used to solve a traffic engineering problem in [43]. Our work can be viewed as an application of this idea to network congestion control.

\section{SUMMARY}

In this paper, we propose VCP, a simple, low-complexity congestion control protocol for high BDP networks. Using extensive ns2 simulations, we show that VCP achieves high utilization, reasonable fairness, low persistent bottleneck queue, and negligible packet loss rate. VCP achieves all these desirable properties while requiring only two bits to encode the network congestion information. Since it can leverage the two ECN bits to carry this information, VCP requires no changes of the IP header. In this respect, VCP can be seen as an extension of the TCP + AQM/ECN proposals that scales to high BDP networks.
To better understand the behavior of VCP, we propose a fluid model, and use this model to analyze the efficiency, fairness, and convergence properties of a simplified version of VCP. Particularly, we prove that the model is globally stable for the case of a single bottleneck link shared by long-lived flows with identical RTTs.

As future work, it would be interesting to study what improvements are possible in VCP by using more than two bits for the congestion-related information. One obvious possibility would be to use a finer granularity encoding of the network load factor to improve the fairness convergence speed. While in this paper we evaluate VCP through extensive simulations, ultimately, only a real implementation and deployment will allow us to asses the strengths and limitations of VCP.

\section{ACKNOWLEDGEMENTS}

The authors are very grateful to Sally Floyd, Dina Katabi, K. K. Ramakrishnan, Scott Shenker and the anonymous reviewers for their insightful comments, and to Dina Katabi for shepherding this paper. The authors owe their gratitude to Jianghai $\mathrm{Hu}$ and John Wen for proof-reading Theorem 1, to Xinzhe Fan and Yang Kuang for their suggestions on the stability analysis, to Dilip Anthony Joseph and Jayanthkumar Kannan for reading earlier drafts of this paper, to David Harrison for making his ns2 graphing tools available, and to Lan Shi for her help. We would like to thank them all.

\section{REFERENCES}

[1] M. Allman, V. Paxson, and W. Stevens. TCP Congestion Control. IETF RFC 2581, April 1999.

[2] S. Athuraliya, V. Li, S. Low, and Q. Yin. REM: Active Queue Management. IEEE Network, 15(3):48-53, May 2001.

[3] D. Bansal and H. Balakrishnan. Binomial Congestion Control Algorithms. INFOCOM'01, April 2001.

[4] D. Bertsekas and R. Gallager. Data Networks. 2nd Ed., Simon \& Schuster, December 1991.

[5] S. Bhandarkar, S. Jain, and A. Reddy. Improving TCP Performance in High Bandwidth High RTT Links Using Layered Congestion Control. PFLDNet'05, February 2005.

[6] L. Brakmo and L. Peterson. TCP Vegas: End to End Congestion Avoidance on a Global Internet. IEEE J. Selected Areas in Communications, 13(8):1465-1480, October 1995.

[7] H. Bullot and R. Les Cottrell. Evaluation of Advanced TCP Stacks on Fast Long-Distance Production Networks. Available at http://www.slac.stanford.edu/grp/scs/net/talk03/tcp-slacnov03.pdf.

[8] C. Casetti, M. Gerla, S. Mascolo, M. Sansadidi, and R. Wang. TCP Westwood: End-to-End Congestion Control for Wired/Wireless Networks. Wireless Networks Journal, 8(5):467-479, September 2002.

[9] A. Charny, D. Clark, and R. Jain. Congestion Control with Explicit Rate Indication. IEEE ICC'95, June 1995.

[10] D. Chiu and R. Jain. Analysis of the Increase/Decrease Algorithms for Congestion Avoidance in Computer Networks. J. of Computer Networks and ISDN, 17(1):1-14, June 1989.

[11] M. Crovella and A. Bestavros. Self-Similarity in World Wide Web Traffic: Evidence and Possible Causes. IEEE/ACM Trans. Networking, 5(6):835-846, December 1997.

[12] S. Deb and R. Srikant. Global Stability of Congestion Controllers for the Internet. IEEE Trans. Automatic Control, 48(6):1055-1060, June 2003.

[13] A. Durresi, M. Sridharan, C. Liu, M. Goyal, and R. Jain. Multilevel Explicit Congestion Notification. SCI'01, July 2001.

[14] W. Feng, K. Shin, D. Kandlur, and D. Saha. The BLUE active queue management algorithms. IEEE/ACM Trans. Networking, 10(4):513-528, August 2002.

[15] S. Floyd. HighSpeed TCP for Large Congestion Windows. IETF RFC 3649, December 2003. 
[16] S. Floyd, M. Handley, J. Padhye, and J. Widmer. Equation-Based Congestion Control for Unicast Applications. SIGCOMM'O0, August 2000

[17] S. Floyd and T. Henderson. The NewReno Modification to TCP's Fast Recovery Algorithm. IETF RFC 2582, April 1999.

[18] S. Floyd and V. Jacobson. Random Early Detection Gateways for Congestion Avoidance. IEEE/ACM Trans. Networking, 1(4):397-413, August 1993.

[19] S. Floyd and V. Paxson. Difficulties in Simulating the Internet. IEEE/ACM Trans. Networking, 9(4):392-403, August 2001.

[20] E. Gafni and D. Bertsekas. Dynamic Control of Session Input Rates in Communication Networks. IEEE Trans. Automatic Control, 29(11):1009-1016, November 1984.

[21] R. Gibbens and F. Kelly. Resource Pricing and the Evolution of Congestion Control. Automatica, 35:1969-1985, 1999.

[22] K. Gopalsamy. Stability and Oscillations in Delay Differential Equations of Population Dynamics. Kluwer Academic Publishers, 1992.

[23] C. Hollot, V. Misra, D. Towlsey, and W. Gong. On Designing Improved Controllers for AQM Routers Supporting TCP Flows. INFOCOM'01, April 2001.

[24] C. Hollot, V. Misra, D. Towsley, and W. Gong. Analysis and Design of Controllers for AQM Routers Supporting TCP Flows IEEE Trans. Automatic Control, 47(6):945-959, June 2002.

[25] V. Jacobson. Congestion Avoidance and Control. SIGCOMM'88, August 1988.

[26] A. Jain and S. Floyd. Quick-Start for TCP and IP. IETF Internet Draft draft-amit-quick-start-02.txt, October 2002.

[27] R. Jain, S. Kalyanaraman, and R. Viswanathan. The OSU Scheme for Congestion Avoidance in ATM Networks: Lessons Learnt and Extensions. Performance Evaluation, 31(1):67-88, November 1997.

[28] R. Jain and K. K. Ramakrishnan. Congestion Avoidance in Computer Networks with A Connectionless Network Layer: Concepts, Goals, and Methodology. Proc. IEEE Computer Networking Symposium, April 1988.

[29] R. Jain, K. K. Ramakrishnan, and D. Chiu. Congestion Avoidance in Computer Networks with a Connectionless Network Layer. DEC-TR-506, August 1987.

[30] H. Jiang and C. Dovrolis. Passive Estimation of TCP Round-Trip Times. ACM Computer Communications Review, $32(3): 75-88$, July 2002.

[31] C. Jin, D. Wei, and S. Low. FAST TCP: Motivation, Architecture, Algorithms, Performance. INFOCOM'04, March 2004

[32] R. Johari and D. Tan. End-to-End Congestion Control for the Internet: Delays and Stability. IEEE/ACM Trans. Networking, 9(6):818-832, December 2001.

[33] L. Kalampoukas, A. Varma, and K. K. Ramakrishnan. Dynamics of an Explicit Rate Allocation Algorithm for Available Bit-Rate (ABR) Service in ATM Networks. Proceedings of the IFIP/IEEE Conference on Broadband Communications, April 1996.

[34] S. Kalyanaraman, R. Jain, S. Fahmy, R. Goyal, and B. Vandalore. The ERICA Switch Algorithm for ABR Traffic Management in ATM Networks. IEEE/ACM Trans. Networking, 8(1), February 2000.

[35] D. Katabi, M. Handley, and C. Rohrs. Congestion Control for High Bandwidth-Delay Product Networks. SIGCOMM'02, August 2002.

[36] T. Kelly. Scalable TCP: Improving Performance in Highspeed Wide Area Networks. Submitted, December 2002.

[37] F. Kelly, A. Maulloo, and D. Tan. Rate Control in Communication Networks: Shadow Prices, Proportional Fairness and Stability. Journal of the Operational Research Society, 49:237-252, 1998.

[38] A. Kesselman and Y. Mansour. Adaptive TCP Flow Control. PODC'03, July 2003.

[39] Y. Kuang. Delay Differential Equations with Applications in Population Dynamics. Academic Press, 1993.

[40] H. Kung, T. Blackwell, and A. Chapman. Credit-Based Flow Control for ATM Networks: Credit Update Protocol, Adaptive Credit Allocation, and Statistical Multiplexing. SIGCOMM'94, August 1994.

[41] S. Kunniyur and R. Srikant. End-To-End Congestion Control: Utility Functions, Random Losses and ECN Marks. INFOCOM'O0, March 2000.

[42] S. Kunniyur and R. Srikant. Analysis and Design of an Adaptive Virtual Queue (AVQ) Algorithm for Active Queue Management. SIGCOMM'01, August 2001.
[43] C. Lagoa, H. Che and B. Movsichoff. Adaptive Control Algorithms for Decentralized Optimal Traffic Engineering in the Internet. IEEE/ACM Trans. Networking, 12(3):415-428, June 2004.

[44] T. Lakshman and U. Madhow. The Performance of TCP/IP for Networks with High Bandwidth-delay Products and Random Loss. IEEE/ACM Trans. Networking, 5(3):336-350, June 1997.

[45] D. Leith and R. Shorten. H-TCP: TCP for High-speed and Long-distance Networks. PFLDnet'04, February 2004.

[46] W. Leland, M. Taqqu, W. Willinger, and D. Wilson. On the Self-Similar Nature of Ethernet Traffic. SIGCOMM'93, August 1993.

[47] D. Lin and R. Morris. Dynamics of Random Early Detection. SIGCOMM'97, August 1997.

[48] S. Low and D. Lapsley. Optimization Flow Control, I: Basic Algorithm and Convergence. IEEE/ACM Trans. Networking, 7(6):861-875, December 1999.

[49] S. Low, F. Paganini, J. Wang, and J. Doyle. Linear Stability of TCP/RED and a Scalable Control. Computer Networks Journal, 43(5):633-647, December 2003.

[50] L. Massoule. Stability of Distributed Congestion Control with Heterogeneous Feedback Delays. IEEE Trans. Automatic Control, 47(6):895-902, June 2002.

[51] M. Mathis, J. Mahdavi, S. Floyd, and A. Romanow. TCP Selective Acknowledgement Options. IETF RFC 2018, October 1996.

[52] Network Simulator ns-2. Http://www.isi.edu/nsnam/ns/.

[53] J. Padhye, V. Firoiu, D. Towsley, and J. Kurose. Modeling TCP Throughput: A Simple Model and its Empirical Validation. SIGCOMM'98, September 1998.

[54] R. Pan, K. Psounis, and B. Prabhakar. CHOKe, A Stateless Active Queue Management Scheme for Approximating Fair Bandwidth Allocation. INFOCOM'O0, March 2000.

[55] V. Paxson. End-to-End Internet Packet Dynamics. SIGCOMM'97, September 1997.

[56] V. Paxson and S. Flyod. Wide-Area Traffic: The Failure of Poisson Modeling. SIGCOMM'94, August 1994.

[57] K. K. Ramakrishnan and S. Floyd. The Addition of Explicit Congestion Notification (ECN) to IP. IETF RFC 3168, September 2001.

[58] K. K. Ramakrishnan and R. Jain. A Binary Feedback Scheme for Congestion Avoidance in Computer Networks. SIGCOMM'88, August 1988.

[59] I. Rhee and L. Xu. CUBIC: A New TCP-Friendly High-Speed TCP Variant. PFLDNet'05, February 2005.

[60] I. Stoica, S. Shenker, and H. Zhang. Core-Stateless Fair Queueing: Achieving Approximately Fair Bandwidth Allocations in High Speed Networks. SIGCOMM'98, September 1998.

[61] V. Utkin. Variable Structure Systems with Sliding Modes. IEEE Trans. Automatic Control, 22(2):212-222, April 1977.

[62] G. Vinnicombe. On the Stability of End-to-end Congestion Control for the Internet. Univ. of Cambridge Tech Report CUED/F-INFENG/TR.398, December 2000.

[63] J. Wen and M. Arcak. A Unifying Passivity Framework for Network Flow Control. INFOCOM'03, March, 2003.

[64] E. Wright. A Non-linear Difference-Differential Equation. J. Reine Angew. Math., 494:66-87, 1955.

[65] B. Wydrowski and M. Zukerman. MaxNet: A Congestion Control Architecture for Maxmin Fairness. IEEE Comm. Letters, 6(11):512-514, November 2002.

[66] Y. Xia, L. Subramanian, I. Stoica, and S. Kalyanaraman. One More Bit is Enough. UC Berkeley Tech Report, June 2005.

[67] L. Xu, K. Harfoush, and I. Rhee. Binary Increase Congestion Control (BIC) for Fast Long-Distance Networks. INFOCOM'04, March 2004.

[68] Y. Yang and S. Lam. General AIMD Congestion Control. ICNP'00, November 2000.

[69] L. Ying, G. Dullerud, and R. Srikant. Global Stability of Internet Congestion Controllers with Heterogeneous Delays. Proc. American Control Conference, June 2004.

[70] Y. Zhang, S. Kang, and D. Loguinov. Delayed Stability and Performance of Distributed Congestion Control. SIGCOMM'04, September 2004. 